\title{
3D Deconvolution for Cryo-Scanning Transmission Electron Tomography
}

\author{
Barnali Waugh ${ }^{1}$, Sharon G. Wolf ${ }^{2}$, Sarah Rubin ${ }^{1}$, Eric Branlund ${ }^{3}$, Zvi Kam ${ }^{4}$, John Sedat ${ }^{3}$ and Michael
} Elbaum $^{1}$

${ }^{1 .}$ Dept of Materials and Interfaces, Weizmann Institute of Science, Rehovot, Israel

2. Dept of Chemical Research Support, Weizmann Institute of Science, Rehovot, Israel

3. Dept of Biochemistry \& Biophysics, Univ of California, San Francisco, California, USA

${ }^{4 .}$ Dept of Molecular Cell Biology, Weizmann Institute of Science, Rehovot, Israel

Electron cryo-tomography is the premier technique for study of cell structure in three dimensions. However, the implementation of conventional transmission EM based on phase contrast is limited to samples thinner than most cells of interest. The scanning transmission mode, STEM, offers significant advantages for tomography of thick specimens [1,2]. These include a longer depth of focus, dynamic focusing at high tilts, and relative immunity to image degradation by inelastic scattering processes. Small convergence angles provide a large depth of field, albeit with some compromise in resolution. In practice, cryo-tomography by STEM (CSTET) reaches a useful specimen thickness in excess of 1 micron.

Similarly to conventional TEM cryo-tomography, CSTET suffers from artifacts inherent to the tilt geometry, in particular, a discrete number of projections acquired over a limited range of angles. The limited range leads to a so-called missing wedge in Fourier space. Three dimensional reconstructions generated from this incomplete set of original data include a number of characteristic distortions, including elongation, poor closure of surfaces, and projection of "ghost" contrast from dense objects into adjacent planes along the optic axis.

Qualitatively, the "ghost" artifacts in electron tomography recall the haze that appears in wide-field optical fluorescence microscopy from out-of-focus sources. Computational deconvolution is an effective means to remove this haze by reassigning diffuse intensity back to the source in a different plane [3]. We asked whether this approach could be applied effectively to 3D cryo-STEM tomograms. In contrast to the light microscope, EM involves only very small angles from the axis (equivalent numerical apertures $10^{-2} \sim 10^{-3}$ ). In STEM tomography the illumination profile within the specimen is typically rod-shaped in each projection. 3D reconstruction involves a back-projection from all the acquired angles, leading to an effective point spread function (PSF) with one rod per tilt, i.e., a fan of spines in the plane perpendicular to the tilt axis. This synthetic PSF is very different from the conical profile relevant to optical epi-fluorescence microscopy, but recalls the $3 \mathrm{D}$ contrast transfer function used in correction of conventional cryo-tomograms for sub-tomogram averaging [4].

We show that CSTET reconstructions can indeed be improved by methods developed for fluorescence deconvolution, using the appropriate PSF. Out-of-plane projections are suppressed, and with them much of the "criss-cross" noise that appears in distant planes. The contrast improvement is demonstrated using gold nanoparticles (Fig 1) and cellular tomograms (Fig 2). CSTET deconvolution offers another step toward whole-cell cryo-tomography at macromolecular resolution. 
References:

[1] SG Wolf, L Houben, M Elbaum, Nat. Methods. 11 (2014), p. 423.

[2] M Elbaum, SG Wolf, L Houben, Bull. 41 (2016), p. 542.

[3] M. Arigovindan et al, Proc. Natl. Acad. Sci. U. S. A. 110 (2013), p. 17344.

[4] TAM Bharat et al, Structure. 23 (2015), p. 1743.
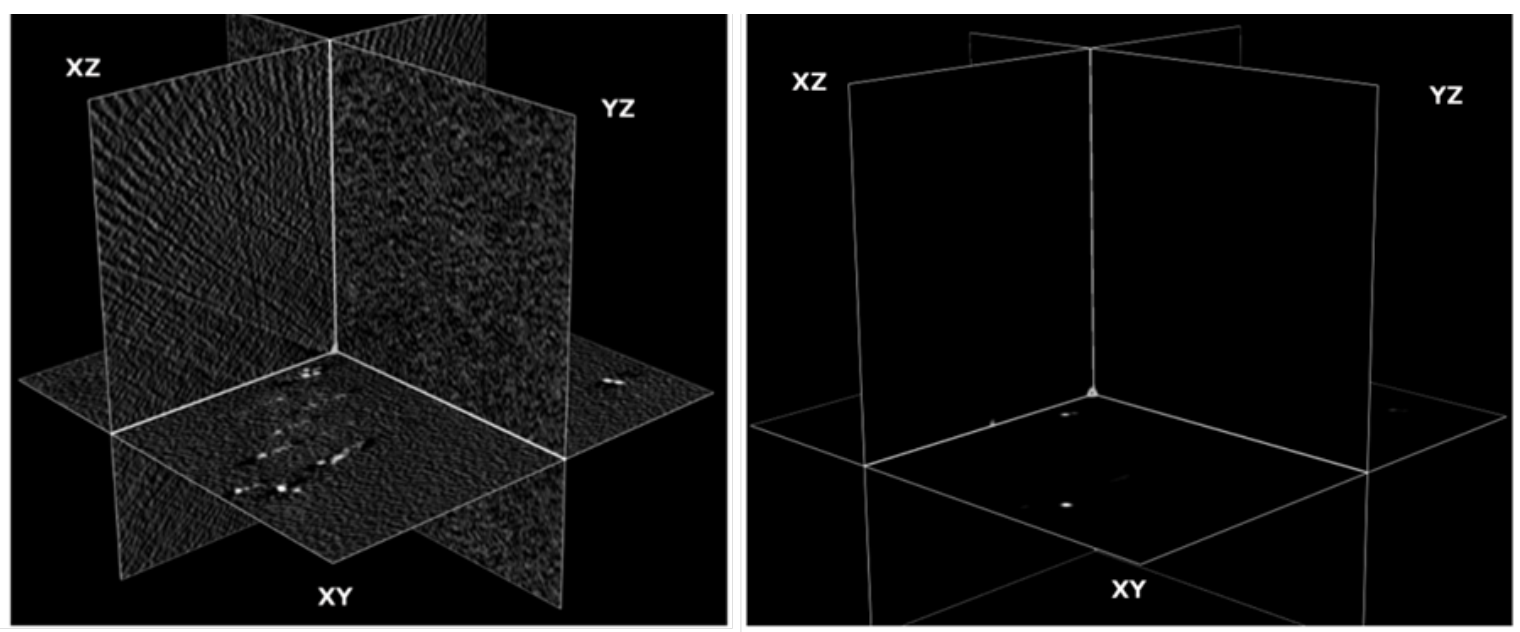

Figure 1. Gold nanoparticles $(6 \mathrm{~nm})$ were suspended in water and vitrified for cryo-tomography. The figure shows orthoslices through a 3D volume reconstructed by weighted back-projection before (left) and after (right) deconvolution using the ER-Decon II algorithm [3].
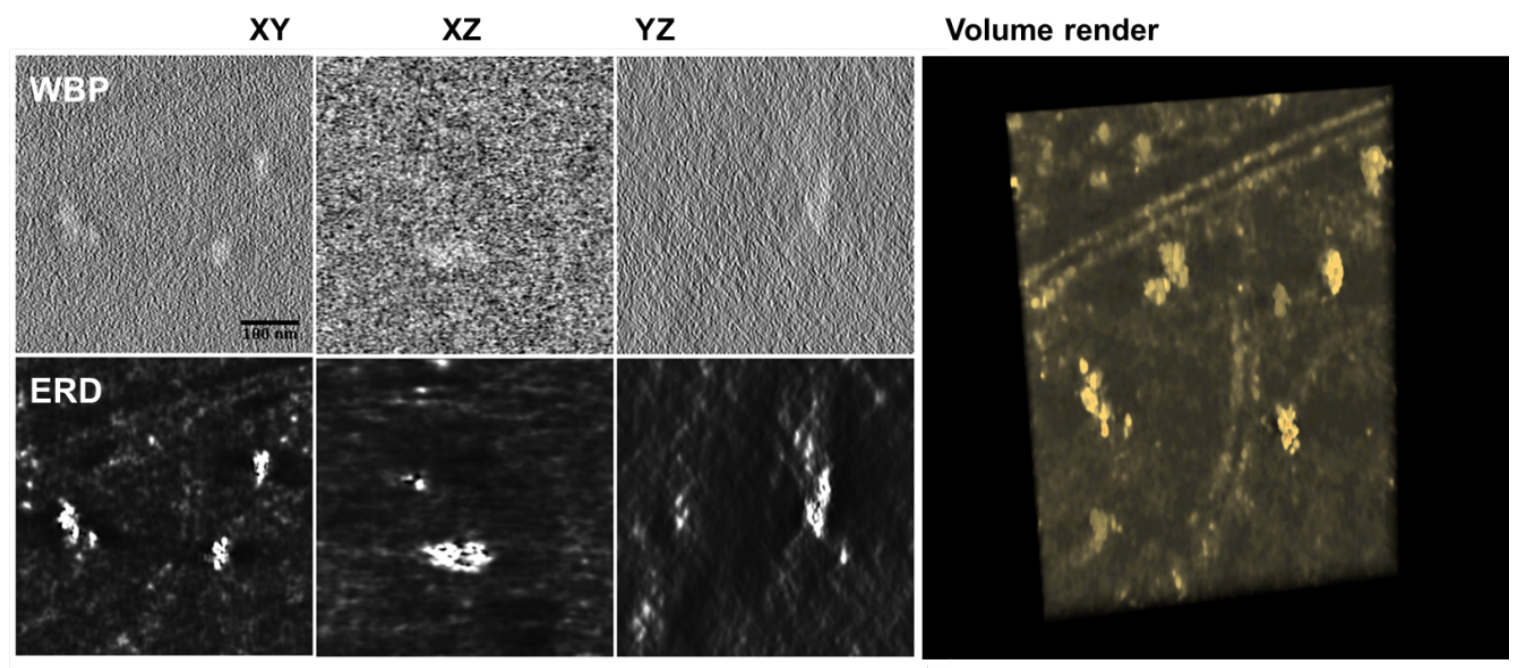

Figure 2. Mitochondrial matrix granules were recorded in CSTET. Tilt series data were reconstructed by weighted back projection (WBP), and further processed by classic iterative deconvolution (ID) [4] or by entropy-regularized deconvolution (ERD) [3]. scale bar $100 \mathrm{~nm}$. A volume rendering of the ERD shows that both high and low contrast features are well restored. 